\title{
Investigating the Effect of Artificial Ageing on the Creep and Recovery of SBS-Modified Bitumen
}

\author{
Giacomo Cuciniello, ${ }^{1, *}$, Pietro Leandri ${ }^{2}$, Davide Lo Presti ${ }^{1}$, Massimo Losa $^{2}$, and Gordon Airey ${ }^{1}$ \\ ${ }^{1}$ Nottingham Transportation Engineering Centre (NTEC), University of Nottingham, Nottingham NG7 2RD, UK \\ ${ }^{2}$ Department of Civil and Industrial Engineering, University of Pisa, Largo Lucio Lazzarino, 56122 Pisa, PI, Italy
}

\begin{abstract}
Polymer additives are widely used to improve the performances of road bitumen and mixtures including their oxidative resistance. However, their effect on the oxidative mechanism remains unclear. This study includes the investigation of the changes in the rheological response of Polymer Modified Bitumen (PMBs) prepared with the Styrene Butadiene Styrene (SBS) copolymer and Sulphur as cross-linker. The bitumen samples were aged in the Rolling Thin Film Oven (RTFO) and in multiple cycles of Pressure Aging Vessel (PAV). The rheological properties were measured by means of the Multiple Stress Creep and Recovery (MSCR) test. Results show that in the case of cross-linked SBS modified bitumen the polymer phase mitigates the effect of hardening of the bitumen phase on the rheological response as a function of the polymer concentration. Adversely, in the SBS modified bitumen without cross-linker, the effect of the polymer vanishes after the RTFO.
\end{abstract}

\section{Introduction}

Polymer additives are widely used to enhance the properties of road bitumen and mixtures [1-5]. Their usage improves the mechanical response of asphalt mixtures including their durability. Besides the enhancement of the mechanical properties, polymer modified bitumen (PMBs) are expected to be stable at storage and ageing resistant [4]. However, if the oxidative mechanism of unmodified bitumen is relatively clear, in the case of PMBs the effect of polymers on the bitumen oxidative ageing needs to be investigated [4-6].

In the case of unmodified bitumen, the oxygen uptake causes an increase in the polar fractions (i.e., asphaltenes) characterized by stronger molecular interactions and a reduced molecular mobility. This mechanism results macroscopically in a reduced strain tolerance of the bitumen with the mixture being more prone to crack at intermediate and low temperatures [810]. In the case of PMBs, a unique ageing mechanism cannot be identified due to the variability in the composition of polymer additives and to the complexity of their interactions with the bitumen phase [2, 3]. However, the study of ageing in PMBs is not recent and some general conclusions can be drawn for specific polymer additives. In the case of Styrene-ButadieneStyrene (SBS) modified bitumen, the polymer phase undergoes thermo-oxidative degradation displaying chain scission and cross-linking. On the other hand, the bitumen phase seems to harden as observed in unmodified bitumen. The polymer degradation appears to mitigate the hardening of the bitumen inducing a more viscous effect on the mechanical response [7, 11-19].
However, the majority of the studies lack details on the constitution of PMBs with solely the polymer type and concentration being available. For instance, information on the usage of cross-linking agents are not provided. Furthermore, the rheological characterization of aged PMBs is generally conducted in the linear viscoelastic region at very low strain levels. The limitations of the linear viscoelastic characterization of PMBs have been discussed in details by several authors $[20,21-23,27,28]$. One of the main concerns is that the reduced strain level applied is not sufficient to determine the stress dependency of polymers because the polymer network is not sufficiently elongated [23]. Therefore, the effect of the thermo-oxidative degradation of the polymer chains on the mechanical response of PMBs needs to be investigated at higher strain levels. Nevertheless, this approach has not been well considered so far $[6,26]$.

The Multiple Stress Creep and Recovery (MSCR) test was specifically developed for polymer modified bitumen to apply stress (and strain) levels capable of mobilizing the polymer chains to account for the stress dependency and the non-linearity in the response of PMBs [23, 24].

This paper aims at investigating the effects of artificial ageing on different SBS modified bitumen prepared by varying the presence of Sulphur as a crosslinking agent. Multiple levels of laboratory-simulated ageing are applied to the different SBS modified bitumen, which are characterized in the non-linear viscoelastic range by the MSCR test. The values of the non-recoverable compliance $\left(\mathrm{J}_{\mathrm{nr}}\right)$ and on the average percent recovery $(\mathrm{R})$, measured at a stress level of 3.2

\footnotetext{
* Corresponding author: evxgc3@nottingham.ac.uk
} 
$\mathrm{kPa}$ have been considered as rheological indicators of the bitumen hardening and of the polymer degradation. Specifically, the $J_{\mathrm{nr}}$ is representative of the overall resistance to the accumulation of permanent deformation of the system bitumen + polymer. Differently, the average percent recovery $(\mathrm{R})$ is considered an indicator of the quality of modification and of the integrity of the polymer network through ageing $[6,24,25]$.

\section{Materials and test methods}

\subsection{Materials}

Six types of bitumen were prepared by combining one base bitumen (Penetration grade - Pen 70/100) with a radial SBS copolymer (polystyrene content 29-31\%), and sulphur (S) used as a cross-linker. The bitumen types are given in Table 1 .

Table 1. Bitumen used in the experiment.

\begin{tabular}{|c|c|c|c|c|c|}
\hline \multirow{2}{*}{ Bitumen } & \multirow[t]{2}{*}{ Base } & \multicolumn{2}{|c|}{$\begin{array}{c}\text { Concentration, } \\
\%\end{array}$} & \multirow{2}{*}{$\begin{array}{l}\text { Mixing } \\
\text { Temp., } \\
{ }^{\circ} \mathrm{C}\end{array}$} & \multirow{2}{*}{$\begin{array}{c}\text { Mixing } \\
\text { time, } \\
\text { min }\end{array}$} \\
\hline & & SBS & Sulphur & & \\
\hline $\begin{array}{c}\text { Pen } 70- \\
100 \text { (Pen) }\end{array}$ & - & - & - & - & - \\
\hline Pen/S & \multirow{6}{*}{$\begin{array}{c}\text { Pen } 70- \\
100\end{array}$} & 0 & 0.1 & \multirow{6}{*}{180} & \multirow{6}{*}{180} \\
\hline SBS 2/S & & 2 & 0.1 & & \\
\hline SBS 4/S & & 4 & 0.1 & & \\
\hline SBS 4 & & 4 & 0 & & \\
\hline SBS 6/S & & 6 & 0.1 & & \\
\hline SBS 6 & & 6 & 0 & & \\
\hline
\end{tabular}

Note: SBS $2 / \mathrm{S}$ (2\%SBS + $0.1 \%$ sulphur); SBS $4 / \mathrm{S}(4 \%$ SBS $+0.1 \%$ sulphur); SBS $6 / \mathrm{S}(6 \%$ SBS $+0.1 \%$ sulphur) SBS 4 (4\%SBS no sulphur); SBS (6\%SBS no sulphur).

The SBS modified bitumens were prepared by using a Silverson ${ }^{\circledR}$ high shear laboratory mixer. In the case of SBS bitumens with sulphur, the bitumen was heated to $180^{\circ} \mathrm{C}$ and the SBS polymer was added to the mix that was subsequently blended at high shear (5,000 RPM) for 1 hour. Afterwards, $0.1 \%$ of sulphur by weight of the modified bitumen was added to the mix and the rotations were reduced approximately to 2,800 RPM for two additional hours. For the SBS modified bitumens without sulphur, the same process was followed with the only difference that sulphur was not used. In the case of the sulphur additivated bitumen $(\mathrm{Pen} / \mathrm{S})$, the same process was followed with the difference that after 1 hour only $0.1 \%$ of sulphur by weight was added to the bitumen

\subsection{Laboratory simulated ageing}

The bitumens were laboratory short-term aged in the Rolling Thin Film Oven (RTFO) and long-term aged in the Pressure Ageing Vessel (PAV) by following the AASHTO R28. Besides the traditional PAV exposure of 20 hours, additional cycles of PAV were used (i.e., 40 hours - 2 PAV; 80 hours -4 PAV).

\subsection{Multiple stress creep and recovery (MSCR)}

The MSCR was conducted at two stress levels (i.e., 0.1 $\mathrm{kPa}$ and $3.2 \mathrm{kPa}$ ) as per the AASHTO $\mathrm{T} 350$. At each stress level, 10 creep and recovery cycles were applied. The values of $\mathrm{J}_{\mathrm{nr}}$ and $\mathrm{R}$ were calculated at each loading cycle and then averaged at each stress level.

The non-recoverable compliance $\left(\mathrm{J}_{\mathrm{nr}}\right)$ at the $\mathrm{n}$-cycle was calculated using Equation (1).

$$
J_{n r}^{n}\left(k P a^{-1}\right)=\frac{\varepsilon_{r}^{n}-\varepsilon_{0}^{n}}{\tau_{0}}
$$

Where:

- $\quad \varepsilon_{r}^{n}$ is the strain value at the end of the recovery phase;

- $\quad \varepsilon_{0}^{n}$ is the initial strain value at the beginning of the creep portion;

- $\tau_{0}$ is the value of the stress level used in the loading cycle.

The percent recovery (R) at the n-cycle was calculated using Equation (2).

$$
R^{n}(\%)=\frac{\varepsilon_{c}^{n}-\varepsilon_{r}^{n}}{\varepsilon_{c}^{n}-\varepsilon_{0}^{n}}
$$

Where:

- $\varepsilon_{c}^{n}$ is the strain value at the end of the creep portion.

The results incorporated in this paper refer to the stress level of $3.2 \mathrm{kPa}$ since at such a stress level the polymer chains should start showing slippage in the test [23]. The MSCR testing temperatures (Table 2) were selected as the temperature at which the parameter $\mathrm{G}^{*} / \sin \delta$ measured on the RTFO residue is equal to 2.20 $\mathrm{kPa}$ (defined as the Continuous Grade High Temperature).

Table 2. MSCR test temperatures.

\begin{tabular}{|c|c|}
\hline Bitumen & MSCR Temperature, ${ }^{\circ} \mathbf{C}$ \\
\hline Pen & 64 \\
\hline Pen/S & 64 \\
\hline SBS 2/S & 70 \\
\hline SBS 4/S & 75 \\
\hline SBS 4 & 75 \\
\hline SBS 6/S & 87 \\
\hline SBS 6 & 87 \\
\hline
\end{tabular}

The MSCR test is conducted at the high PG temperature on the RTFO residue only since rutting is observed to occur at early stage in the pavement life. This work aims to evaluate the changes in the creep and recovery after the short-term ageing and after multiple levels of PAV exposures. Therefore, the MSCR test temperatures were determined on the RTFO residue and then maintained constant to highlight the effects of the PAV only avoiding any possible confounding effect. In some measure, this is representative of the field conditions. That is, the bitumen is selected as per its high 
temperature properties determined after the short-term ageing. Then, as per the criterion adopted, it is possible to highlight how these properties (measured at the same temperature) vary with the oxidative ageing simulated in the PAV.

The continuous grade temperature was determined on the SBS modified bitumen including Sulphur (i.e., SBS 2/S, SBS 4/S, SBS 6/S) and on the base bitumen (Pen). For the remaining bitumen (i.e., SBS 4, SBS 6) the MSCR test temperatures correspond to the ones determined on the $\mathrm{PMB} / \mathrm{S}$ bitumen with the same polymer concentration. For the bitumen $\mathrm{Pen} / \mathrm{S}$, the MSCR temperature corresponds to the one determined on the Pen bitumen. It is worth clarifying that according to AASHTO M 332, the traffic designation should be measured at the Performance Grade temperature and not at the Continuous Grade High Temperature. However, the traffic designation is included to provide a synthetic indicator of the change in the resistance to the accumulation of permanent deformation and not for grading purpose. Three replicates were used per each bitumen.

\section{Results and discussion}

Prior to discuss the results of the Jnr and the average percent recovery at different levels of ageing, two examples of the trend of the measured strain versus time in the MSCR test are given. Specifically, the figures below show variation of the measured strain (at $3.2 \mathrm{kPa}$ ) versus time of the unmodified bitumen (Pen - Figure 1) and the SBS 6/S modified bitumen (Figure 2).

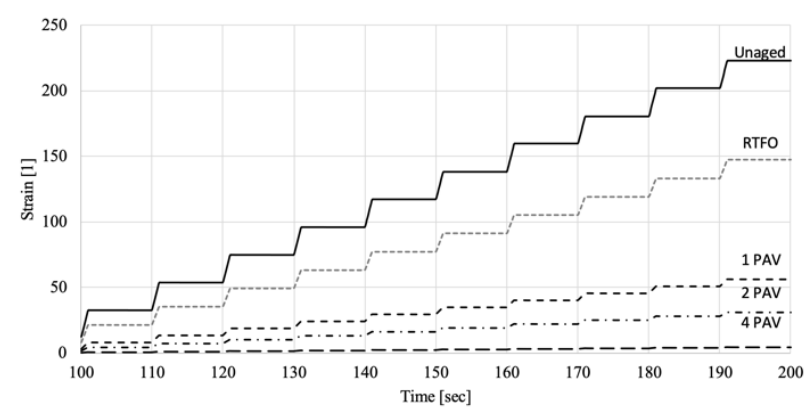

Fig. 1. Measured strain at $3.2 \mathrm{kPa}$ versus time Pen bitumen at different levels of ageing.

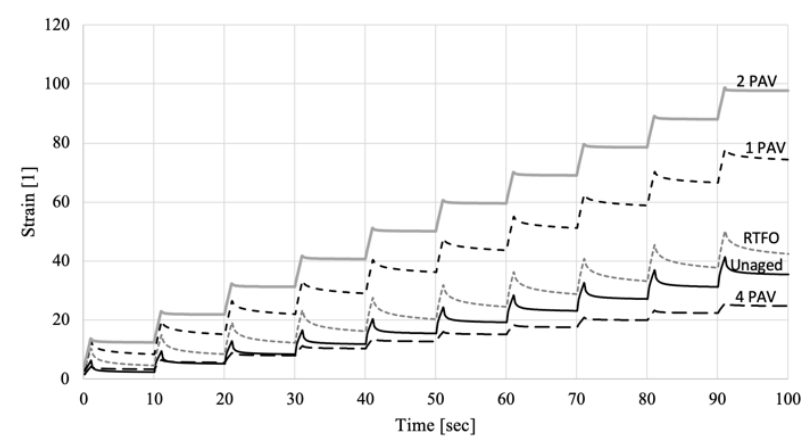

Fig. 2. Measured strain at $3.2 \mathrm{kPa}$ versus time SBS $6 / \mathrm{S}$ at different levels of ageing.
In the case of the unmodified bitumen (Figure 1), the accumulated final strain at the end of the loading cycles decreases with ageing with the highest value of final strain at the unaged condition and the lowest after four cycles of PAV. This trend highlights the oxidative hardening of the bitumen occurring in the RTFO and in the PAV. On the other hand, the final accumulated strain of the SBS 6/S bitumen (Figure 2) shows the opposite trend between the unaged and the 2PAV-aged conditions. In this case, the final strain increases with ageing indicating that the bitumen is softening in the RTFO and the PAV. Then, after four cycles of PAV, the final strain reduces below the value shown at the unaged condition. The variation of the final strain with ageing depends on the contributions of the polymer and of the bitumen phases at the testing temperatures. This mechanism is detailed in the discussion of the $\mathrm{J}_{\mathrm{nr}}$ and the $\mathrm{R}$ results.

\subsection{MSCR - Analysis of Jnr values}

The values of the $\mathrm{J}_{\mathrm{nr}}$ at the stress level of $3.2 \mathrm{kPa}$ are given in Figure 3 and discussed as follows.

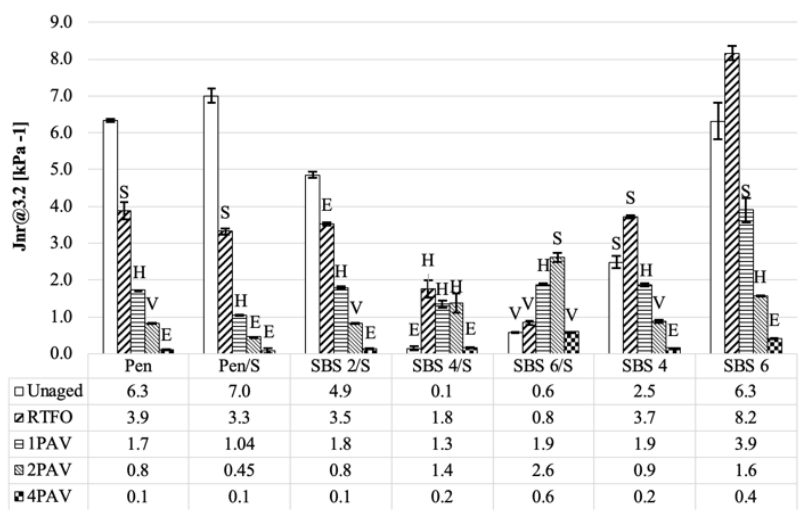

Fig. 3. $\mathrm{J}_{\mathrm{nr}} @ 3.2 \mathrm{kPa}\left(\mathrm{kPa}^{-1}\right)$

(AASHTO M 332 traffic Designation: $\mathrm{S}-$ Standard $\left(\mathrm{J}_{\mathrm{nr}} \max =4.5 \mathrm{kPa}^{-1}\right) ; \mathrm{H}$ - Heavy $\left(\mathrm{J}_{\mathrm{nr}} \max =2 \mathrm{kPa}^{-1}\right) ; \mathrm{V}-$ Very Heavy $\left(\mathrm{J}_{\mathrm{nr}} \max =1 \mathrm{kPa}^{-1}\right) ; \mathrm{E}-$ Extreme $\left.\left(\mathrm{J}_{\mathrm{nr} \max }=0.5 \mathrm{kPa}^{-1}\right)\right)$

\subsubsection{Pen and Pen/S bitumen}

The non-recoverable compliance of the Pen bitumen decreases with ageing. This result is not surprising since the stiffening effect of ageing on unmodified bitumen is well known. The use of $0.1 \%$ Sulphur does not provide a remarkable effect with the $\mathrm{J}_{\mathrm{nr}}$ showing the same trend.

\subsubsection{SBS/S Modified bitumen (SBS + Sulphur)}

For the selected testing temperatures, the variation of the $\mathrm{J}_{\mathrm{nr}}$ values with ageing of the SBS-modified bitumen depends on the polymer concentration.

The $\mathrm{J}_{\mathrm{nr}}$ values of the SBS 2/S bitumen decrease with ageing as observed in the Pen bitumen. It seems that at such polymer concentration the polymer phase is not capable of mitigating the effects on of the bitumen (phase) hardening on the rheological response. 
In the SBS 4/S bitumen, the $\mathrm{J}_{\mathrm{nr}}$ increases after the RTFO to remain within the test variability (H-grade) up to two cycles of PAV. After four cycles of PAV, the $J_{n r}$ values drops drastically with the traffic designation becoming E-grade. It seems that between the RTFO and two PAV cycles, the degradation of the polymer chains compensates for the hardening of the bitumen keeping the $\mathrm{J}_{\mathrm{nr}}$ values constant throughout the oxidation.

Therefore, at the selected testing temperature the mechanical response is controlled by both the bitumen and the polymer phases [17]. The effect of the polymer vanishes after four cycles of PAV when the hardening of the bitumen phase causes a drop in the $\mathrm{J}_{\mathrm{nr}}$ value.

In the SBS 6/S bitumen, the $\mathrm{J}_{\mathrm{nr}}$ increases with ageing with the traffic designation varying from a V-grade (unaged) to an S-grade after 2 cycles of PAV. The majority of the degradation occurs in the PAV, with the RTFO producing a marginal damage. After four cycles of PAV, the $J_{n r}$ value drops as seen in the SBS 4/S binder. At such high SBS concentration, the polymer controls the rheological response measured at the selected test temperature. That is, the increase in the $\mathrm{J}_{\mathrm{nr}}$ values might indicate that, although the bitumen phase hardens, the PMB is softening because its response is more controlled by the polymer phase that is degrading in the PAV. Then, as observed in the SBS $4 / \mathrm{S}$ binder, after four cycles of PAV, the effect of the polymer vanishes because it is highly degraded and the rheological response is dominated by the hardened bitumen. Consequently, the $\mathrm{J}_{\mathrm{nr}}$ value drops. The concept of controlling the rheological response (or dominance) as a function of temperature has been detailed by Airey and Brown [17].

\subsubsection{SBS Modified bitumen (No Sulphur)}

The trend of the $\mathrm{J}_{\mathrm{nr}}$ values of the SBS 4 and SBS 6 bitumen highlight that the use of Sulphur as a crosslinker strongly influences the ageing susceptibility of the PMBs. Both bitumens show an increase in the $\mathrm{J}_{\mathrm{nr}}$ values between the unaged condition and the RTFO. After the RTFO, the trend throughout the PAV ageing coincides with what was observed in the Pen bitumen. Although the SBS is used in the same concentrations of the bitumens modifed with Sulphur (i.e., 4\%, 6\%), the rheological response indicates that after the RTFO the polymer phase is degraded and does not compensate for the hardening of the bitumen phase. The advantage of using Sulphur in PMBs have been determined by various authors that highlight that the crosslinking effect of Sulphur improves polymer stability and enhances the quality of modification $[30,31]$. In this case, results show that the cross-linking effect of Sulphur provides the polymer network with a higher oxidative resistance in the PAV with the SBS balancing the hardening of the bitumen phase.

\subsection{MSCR - Analysis of average percent recovery $(\% R)$}

The values of the percent recovery $(\mathrm{R})$ at the stress level of $3.2 \mathrm{kPa}$ are given in Figure 4 and are discussed as follows.

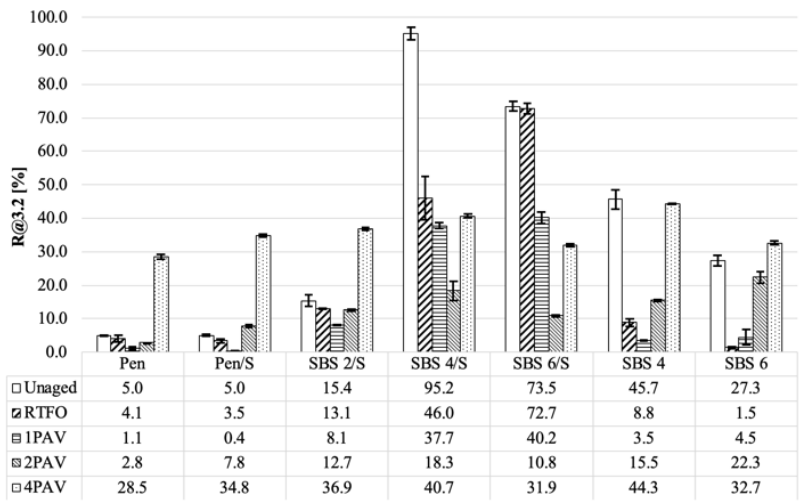

Fig. 4. Percent recovery (R) @ $3.2 \mathrm{kPa}(\%)$.

\subsubsection{Pen and Pen/S bitumen}

The Pen and the Pen/S bitumen are unmodified; therefore, the trend of their percent recovery does not provide any relevant information. However, after four cycles of PAV, an increase in the values of $\mathrm{R}$ of both the bitumen is noted. This aspect might depend on the bitumen hardening that causes a reduction in the creep portion of the strain (Equation (2)).

\subsubsection{SBS/S Modified bitumen (SBS + Sulphur)}

In the SBS modified bitumen with Sulphur, the variation of the percent recovery with ageing $(\mathrm{R})$ is a function of the polymer concentration.

In the SBS 2/S bitumen, the polymer phase produces a marginal increase in the percent recovery compared with the unmodified bitumen. The trend of $\mathrm{R}$ with ageing is similar to those observed in the Pen and the Pen/S bitumen with $\mathrm{R}$ increasing after four cycles of PAV due to the bitumen hardening. As observed with the $\mathrm{J}_{\mathrm{nr}}$ values, values of $\mathrm{R}$ highlight that at $2 \% \mathrm{SBS}$ (and at the selected testing temperature), the response of the PMB with ageing does not deviate from the response of the base bitumen.

In the SBS 4/S bitumen, the polymer modification produces a remarkable increase in the average percent recovery $(\mathrm{R}=95 \%$ at unaged conditions - Figure 4$)$. In the RTFO, the polymer network degrades with the value of $\mathrm{R}$ that drops to $46 \%$. In the PAV, the residual deterioration of the polymer network is visible after 40 hours of exposure with the value of $\mathrm{R}$ decreasing to $18.3 \%$ after two PAV cycles (Figure 4 ). The reduced loss in average percent recovery after a single cycle of PAV (-8\% - Figure 4) is consistent with the trend of the $\mathrm{J}_{\mathrm{nr}}$ values that show a reduced variation. After four cycles of PAV, the hardening of the bitumen phase produces an increase in the $\mathrm{R}$ values as observed in the other bitumen discussed so far (i.e., Pen, Pen/S and SBS 2/S).

The decreasing trend of the percent recovery between the RTFO and two cycles of PAV is an indicator of the 
integrity of the polymer network. The damage accumulated in the polymer network causes the $\mathrm{R}$ values to decrease until the polymer contributes to controlling the PMB response under loaded $\left(\mathrm{J}_{\mathrm{nr}}, \mathrm{R}\right)$ and unloaded conditions (R). Afterwards, once the polymer chains achieve high levels of deterioration, the response is more controlled by the hardened bitumen with the $R$ value increasing.

As observed in the SBS 4/S bitumen, the polymer modification in the SBS 6/S bitumen increases the percent recovery with respect to the unmodified conditions. The SBS 4/S bitumen shows a higher recovery probably because of the difference in the testing temperature (Table 2). The SBS 6/S bitumen maintains almost the same average percent recovery after the RTFO that slightly damages the polymer network (Figure 4). On the other hand, the oxidative process in the PAV degrades the polymer network producing a decrease in percent recovery between the RTFO and two cycles of PAV. The growth in percent recovery after four cycles of PAV depends on the same mechanism detailed for the SBS 4/S bitumen.

\subsubsection{SBS Modified bitumen (No Sulphur)}

Results from the average percent recovery of the SBS 4 and SBS 6 modified bitumen confirm that the use of Sulphur as a cross-linker affects the oxidative resistance of the polymer network measured by means of loss in percent recovery $(\mathrm{R})$. The majority of the degradation of the polymer network occurs in the RTFO with the values of the average percent recovery that drop below $10 \%$ at both concentrations (Figure 4). The trends of the average percent recovery with the PAV exposure do not deviate from the trends of the unmodified bitumen. What should be considered in this case is that both the PMBs contain with the same polymer concentrations of the SBS 4/S and SBS 6/S PMBs and differ only for the presence of Sulphur. Therefore, the effect of the cross-linker on the thermo-oxidative resistance of the polymer network is clearly shown and needs to be considered once the antioxidative properties of SBS polymers are investigated.

\section{Conclusions}

In this study, the effects of laboratory-simulated ageing on the rheological response of SBS-modified bitumen were investigated. Five polymer-modified bitumen were prepared by mixing a Pen $70-100$ base bitumen (Pen) with different concentrations of a radial SBS (polystyrene content 29-31\%) and Sulphur as a crosslinker. Three PMBs were prepared by mixing three concentrations of SBS (i.e., 2\% - medium-low; 4\% high; $6 \%$ - very high) with $0.1 \%$ Sulphur. Two PMBs were prepared by mixing the base bitumen with $4 \%$ and $6 \%$ SBS without cross-linker. One unmodified bitumen was modified with $0.1 \%$ Sulphur $(\mathrm{Pen} / \mathrm{S})$ to isolate any eventual effects of the Sulphur. The bitumen were laboratory-aged combining the RTFO and different times of PAV exposure. The rheological response was measured by the Multiple Stress Creep and Recovery (MSCR) test. The non-recoverable compliance $\left(\mathrm{J}_{\mathrm{nr}}\right)$ and the average percent recovery $(\mathrm{R})$ both measured at 3.2 $\mathrm{kPa}$ were considered as rheological indicators representative of the bitumen hardening and of the polymer degradation $(\mathrm{R})$.

The following concluding remarks can be drawn:

- The $\mathrm{J}_{\mathrm{nr}}$ values of the Pen bitumen decrease with ageing due to the oxidative hardening occurring in the RTFO and in the PAV. At high oxidative levels, the bitumen hardening produces an increase in the average percent recovery measured in the DSR. The bitumen modified with Sulphur (Pen/S) shows the same behavior.

- The rheological response of the cross-linked SBS modified bitumen (i.e., SBS 2/S, SBS 4/S, and SBS $6 / S$ ) varies with ageing depending on the polymer concentration What is observed is that, at the selected test temperatures, the higher the polymer concentration, the more the SBS mitigates the effects of the hardened bitumen phase on the MSCR response.

- At medium-low concentration ( $2 \%)$, the trend of the rheological parameters $\left(\mathrm{J}_{\mathrm{nr}}\right.$ and $\left.\mathrm{R}\right)$ does not deviate from those of the unmodified bitumen.

○ At high concentration (4\%), the polymer network is damaged in the RTFO and the PAV. Nevertheless, between the RTFO and two cycles of PAV, the SBS phase is capable of balancing the hardening of the bitumen phase with the $\mathrm{J}_{\mathrm{nr}}$ values showing limited variation. At higher oxidative levels, the polymer is sensibly damaged and the hardened bitumen phase controls the rheological response with the $\mathrm{J}_{\mathrm{nr}}$ that decreases and the average percent recovery that raises.

$\circ$ At very high SBS concentrations (6\%), the polymer phase controls the rheological response from the unaged conditions up to two cycles of PAV. The polymer network is damaged in the PAV with the RTFO providing negligible effects. Between the RTFO and two cycles of PAV, the $\mathrm{J}_{\mathrm{nr}}$ increases with ageing reversely with what is observed in the unmodified bitumen. At higher oxidative levels, the same behavior of the SBS 4/S bitumen is observed.

- Results show that the use of the average percent recovery as an indicator of integrity of the polymer network can be advocated.

- In the SBS modified bitumens without the crosslinker, the effect of the polymer on the rheological 
response vanishes after the RTFO irrespective of the polymer concentration.

- The combined use of Sulphur and SBS changes the oxidative resistance of PMB. Although the effect of Sulphur cannot be isolated, it seems to be clear that the cross-linked SBS modified bitumens (SBS + Sulphur) have a lower oxidative susceptibility than uncross-linked SBS modifed bitumens with the same polymer concentration. Therefore, in the study of the anti-oxidative properties of SBS, parameters such as the use of the cross-linker need to be considered.

\section{References}

1. Yildirim, Y., Polymer modified asphalt binders. Construction and Building Materials, 21(1), 66-72, (2007).

2. Lesueur, D., The colloidal structure of bitumen: Consequences on the rheology and on the mechanisms of bitumen modification. Advances in Colloid and Interface Science, 145(1-2), 42-82, (2009).

3. Polacco, G., Filippi, S., Merusi, F., and Stastna, G., A Review of the Fundamentals of PolymerModified Asphalts: Asphalt/polymer Interactions and Principles of Compatibility. Advances in Colloid and Interface Science, 224, 72-112, (2015).

4. Bahia H.U., Hanson D.I., Zeng M., Zhai H., Khatri M.A., Anderson M., National Highway Research Program (NCHRP) Report 459 - Characterization of Modified Asphalt Binder in Superpave Mix Design, (2001).

5. Zhu, J., Birgisson, B., and Kringos, N., Polymer modification of bitumen: Advances and challenges. European Polymer Journal, 54, 18-38, (2014).

6. Cuciniello, G., Leandri, P., Filippi, S., Lo Presti, D., Losa, M., and Airey, G.D. Effect of ageing on the morphology and creep and recovery of polymermodified bitumens, Materials and Structures, 51(136), (2018).

7. Goodrich, J.L., Asphalt and Polymer Modified Asphalt Properties Related to the Performance of Asphalt Concrete Mixes (with discussion). Journal of Association of Asphalt Paving Technologists, 57, 116-175, (1988).

8. Petersen, J. C. Chemical Composition of Asphalt as Related to Asphalt Durability: State of the Art. Transportation Research Record 999 Transportation Research Board, 13-30, (1984).

9. Petersen, J., and R. Glaser. Asphalt Oxidation Mechanisms and the Role of Oxidation Products on Age Hardening Revisited. Road Materials and Pavement Design, 12(4), 795-819, (2011).

10. Page, G.C., Murphy, K.H., Ruth, B.E., and Roque, R., Asphalt binder Hardening-Causes and Effects. Journal of Association of Asphalt Paving Technologists, 140-167, (1985).
11. Lu, X., and Isacsson, U., Chemical and Rheological Characteristics of Styrene-Butadiene-Styrene Polymer-Modified Bitumens. Transportation Research Record: Journal of the Transportation Research Board, 1661, 83-92, (1999).

12. Lu, X., and Isacsson, U., Artificial aging of polymer modified bitumens. Journal of Applied Polymer Science, 76(12), 1811-1824, (2000).

13. Xu, J., Zhang, A., Zhou, T., Cao, X., and Xie, Z., A study on thermal oxidation mechanism of styrenebutadiene-styrene block copolymer (SBS). Polymer Degradation and Stability, 92(9), 1682-1691, (2007).

14. Negulescu, I., Muhammad, L., Daly, W., Abadie, C., Cuero, R., Daranga, C., and Glover, I., Chemical and rheological characterization of Wet and Dry Aging of SBS Copolymer Modified Asphalt Cement: Laboratory and Field Evaluation. Journal of the Association of Asphalt Paving Technologists, 75, 267-296, (2006).

15. Lucena, M., Soares, S., and Soares, J., Characterization and thermal behavior of polymermodified asphalt. Materials Research, 7(4), 529-534, (2004).

16. Cortizo, M., Larsen, D., Bianchetto, H., and Alessandrini, J., Effect of the thermal degradation of SBS copolymers during the ageing of modified asphalts. Polymer Degradation and Stability, 86(2), 275-282, (2004).

17. Airey, G.D, and Brown, S. F., Rheological Performance of Aged Polymer Modified Bitumens, Journal of Association of Asphalt Pavement Technologists, 67, 66-100, (1998).

18. J. Oliver, P. Tredera, The Change in Properties of Polymer Modified Binders with Simulated Field Exposure. Journal of the Association of Asphalt Paving Technologists, 66, 570-602, (1997).

19. Lu, X., Soenen, H., Heyrman, S., Redelius, P., Durability of Polymer Modified Binders in Porous Asphalt Pavements, Proceedings of the XXVIII International Baltic Road Conference, (2013).

20. Bahia, H.U., Zhai, H., Onnetti, K., and Kose, S., Non-Linear Viscoelastic and Fatigue Properties of Asphalt Binders. Journal of Association of Asphalt Pavement Technologists, 68, 1-34, (1999).

21. Airey, G. D., Rahimzadeh, B., and Collop, A., Linear Rheological Behavior of Bituminous Paving Materials. Journal of Materials in Civil Engineering, 16(3), 212-220, (2004).

22. Bahia, H.U., Perdomo, D., and Turner, P., Applicability of Superpave Binder Testing Protocols to Modified Binders. Transportation Research Record: Journal of the Transportation Research Board, 1586, 16-23, (1997).

23. D'Angelo, J., Kluttz, R., Revision of the Superpave high temperature binder specification: the multiple stress creep recovery test (with discussion). Journal of the Association of Asphalt Paving Technologists, 76, 123-162, (2007).

24. Golalipour, A., Modification of multiple stress creep recovery test procedure usage in specification. 
Master Thesis, University of Wisconsin at Madison, (2011).

25. Clopotel, C., and Bahia, H.U., Importance of Elastic Recovery in the DSR for Binders and Mastics. Engineering Journal, 16(4), 99-106, (2012).

26. Singh, B., Saboo, N., and Kumar, P., Effect of Short-Term Aging on Creep and Recovery Response of Asphalt Binders. Journal of Transportation Engineering, Part B: Pavements, 143(4), p. 04017017, (2017).

27. Hintz, C., Velasquez, R.A., Li, Z., and Bahia, H.U., Effect of oxidative aging on binder fatigue performance. Journal of the Association of Asphalt Paving Technologists, 80, 527-548, (2011).

28. Cuciniello, G., Leandri, P., Filippi, S., Lo Presti, D., Polacco, G., Losa, M., and Airey, G.D. Microstructure and Rheological Response of Laboratory-Aged SBS-Modified Bitumens. Paper submitted to the 8th EATA Conference, Granada Spain, 3-5 June 2019.

29. Anderson, D.A., Bonaquist, R., National Highway Research Program (NCHRP) Report 709 Investigation of Short-Term Laboratory Aging of Neat and Modified Asphalt binders, (2012).

30. Mandal, T., Sylla, R., Bahia, H.U., and Barmand S., Effect of cross-linking agents on the rheological properties of polymer-modified bitumen. Road Materials and Pavement Design, 16(1), 349-361, (2015).

31. Zhang, F., Yu, J., and $\mathrm{Wu}, \mathrm{S}$., Effect of ageing on rheological properties of storage-stable SBS/sulphur-modified asphalts. Journal of Hazardous Materials, 182(1-3), 507-517, (2010). 